\title{
AMBIGUITY SUPPRESSION BY AZIMUTH PHASE CODING IN MULTICHANNEL SAR SYSTEMS
}

\author{
Federica Bordoni, Marwan Younis, Gerhard Krieger \\ German Aerospace Centre (DLR), Microwaves and Radar Institute, 82234 Wessling, Germany
}

\begin{abstract}
The Azimuth Phase Coding (APC) technique, proposed to suppress range ambiguities in conventional SAR systems, stands out for its low implementation complexity and its effectiveness for point and distributed ambiguities. This paper investigates the possibility of applying the APC to the new, forthcoming generation of multichannel SAR systems, for high resolution and wide swath imaging, based on Digital Beamforming on receive. The extension of APC to multichannel SAR systems is mathematically described. A new metric is defined to quantify the APC performance. A numerical analysis is developed to characterize the influence on the APC behaviors of the main SAR system parameters. Finally, an example of APC performance is provided, by considering two multichannel SAR systems based on a planar and a reflector antenna.
\end{abstract}

Index Terms - SAR, Azimuth Phase Coding, DBF

\section{INTRODUCTION}

The large number of recent and forthcoming spaceborne Synthetic Aperture Radar (SAR) missions for remote sensing, e.g. TerraSAR-X, COSMO-SkyMed, RADARSAT-2, TanDEM-X, Sentinel-1, PAZ, testifies the interest that this sector is currently receiving. Nevertheless, these systems still suffer a basic limitation: high spatial resolution and wide coverage cannot be attained simultaneously. For instance, a resolution around $1 \mathrm{~m}$ can be achieved over a swath width of $10 \mathrm{~km}$; whereas a coverage of $100 \mathrm{~km}$ allows for a resolution in the order of $16 \mathrm{~m}$. The importance for many applications of Earth observation to overcome this limitation has motivated an intensive research. In particular, since the trade-off between spatial resolution and swath width is inherent to the system concept and originated by ambiguity constraints, the research has been oriented in two main directions: i) new, more flexible SAR systems [1-3]; ii) processing methods for removing the ambiguities $[4,5]$.

The new generation of SAR systems is mainly characterized by the use of multiple transmit/receive channels and proper digital processing techniques, such as the Digital Beamforming (DBF) on receive. This allows for a relaxation of the design constraints, a general improvement of the SAR performance and a mitigation of the trade-off between swath width and spatial resolution $[1,2,6]$.

Among the techniques for ambiguity suppression, the one proposed by Dall and Kusk [5], denoted as Azimuth Phase Coding (APC), has recently received particular interest because of its indiscriminate applicability to point and distributed ambiguities, its low implementation complexity, the new degrees of freedom that it offers in the system design $[5,7]$.

The APC technique proposed by Dall and Kusk is conceived for conventional SAR systems. In [7] the advantages offered by APC, in terms of design and imaging modes, are explained by considering conventional systems and multichannel systems with multiple independent receivers. With respect to these systems, a multichannel system could be characterized by additional digital signal processing. Specifically, reconstruction techniques are included, to combine the signals received from the different azimuth channels $[3,6]$. This allows for simultaneous wide swath and high resolution imaging. Nevertheless, the properties of the APC are no more guaranteed.

This paper investigates the behaviors and achievable performance of the APC technique, when applied to a multichannel SAR system based on DBF on receive. As an extension to [7], reconstruction techniques are considered.

\section{REVIEW OF THE AZIMUTH PHASE CODING}

The APC is based on three main steps [5]: (i) azimuth (i.e. pulse to pulse) phase modulation on transmission (Tx); (ii) azimuth phase demodulation on reception (Rx); (iii) azimuth filtering over the processing bandwidth. The modulation/ demodulation phases are chosen such that step (i) and (ii) cancel each other on the useful signal, which is unchanged by APC; whereas the range ambiguous signals are affected by a residual phase, which is linearly dependent on the azimuth sample number, $n[5]$ :

$$
\varphi_{\text {res }}(n, k, M)=\frac{2 \pi}{M} k n, k=0, \pm 1, \pm 2, \cdots
$$

where the sampling interval is the inverse of the pulse repetition frequency (PRF), 1/PRF; $M \geq 2$ is a positive integer, denoted as APC shift-factor. It is worth remarking 
the periodic behavior of $\varphi_{\text {res }}$ versus the order of range ambiguity, $k$. In the frequency domain, the APC produces to a Doppler shift, $\Delta f$, of the range ambiguity of order $k$ :

$$
\Delta f=\Delta f(k, M)=\bmod \left\{k \frac{P R F}{M}\right\}_{P R F / 2},
$$

where $\bmod \{\cdot\}_{P R F / 2}$ denotes the modulus operator, accounting for the periodicity of the Discrete Fourier Transform (DFT) and the limitation within the interval ( $-P R F / 2, P R F / 2]$. As a result, the spectrum of the range ambiguity and that of the useful signal are no more superimposed. Then, in presence of Doppler oversampling, i.e. of a gap between the processed bandwidth and the PRF, the ambiguous power can be filtered out by step (iii).

It is worth noting that $M=2$ maximizes the frequency displacement, $\Delta f=P R F / 2$, between the useful signal and the range ambiguity of $1^{\text {st }}$ order. Consequently, it is reasonable to expect that the best range ambiguity suppression is obtained for $M=2$.

\section{APC IN MULTICHANNEL SAR SYSTEMS BASED ON DIGITAL BEAMFORMING ON RECEIVE}

Consider the multichannel SAR system in Figure 1. It employs a single $\mathrm{Tx}$ and $N \mathrm{Rx}$ azimuth channels. In transmission, the SAR pulse is sent at pulse repetition frequency $P R F$ and a wide azimuth pattern is employed in order to properly cover the desired processed bandwidth, $B_{p}$. In general, $P R F<<B_{p}<<N P R F$. In reception, on each of the $N$ channels, the azimuth signal is sampled at frequency $P R F$. Since $P R F<<B_{p}$, a multichannel reconstruction method should be applied in order to recover the Doppler spectrum over the processed bandwidth. For instance, in [6] a SAR system based on a planar array antenna is considered, where each $\mathrm{Rx}$ channel is associated with a single subaperture and to the whole processed bandwidth. In this case, the azimuth discrete signal on each $\mathrm{Rx}$ channel is strongly aliased and the multi-aperture reconstruction algorithm is applied to obtain an unambiguous multichannel signal. Alternatively, in [3], a reflector-based system is considered, where each $\mathrm{Rx}$ channel is associated with a sharp pattern, covering a sub-band of the overall processing bandwidth. Here, a proper spectral combination of the signals at each channel allows retrieving the overall band of interest [3]. When the APC modulation/demodulation is applied, independent on the specific multichannel reconstruction approach, the azimuth signal on each single $\mathrm{Rx}$ channel experiences the same residual phases and Doppler shift, given by (1) and (2), as in the conventional SAR case. Consequently, after multichannel reconstruction, the APC residual phase on the range ambiguous signal has

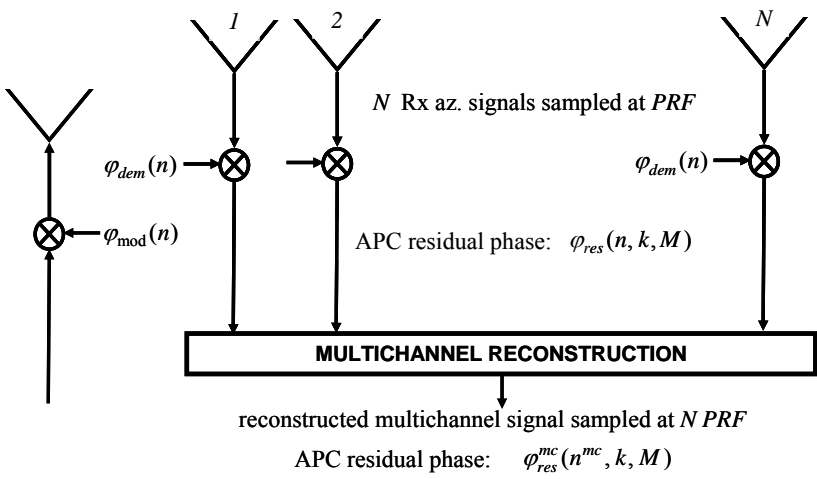

Fig. 1. APC in multichannel SAR system based on DBF on receive.

no more a linear trend versus the azimuth sample number and the properties of APC are no more guaranteed [8]. This assertion could be better explained by considering, without loss of generality, a SAR system based on a planar array antenna, operating with a uniform PRF. Under this hypothesis, the reconstructed multichannel signal is obtained by consecutively interleaving the azimuth samples received by each channel, and is equivalent to the $\mathrm{Rx}$ signal sampled at $P R F_{\text {eff }}=N P R F$, the effective PRF [6]. The APC modulation/demodulation produces the same residual phase, given by (1), on each of the $N$ receivers. Thus, the residual phase on the reconstructed signal has a "stair-shape" [8]:

$\varphi_{\text {res }}^{m c}\left(n^{m c}, k, M\right)=\frac{2 \pi}{M} k \cdot \operatorname{int}\left\{\frac{n^{m c}}{N}\right\}, k=0, \pm 1, \pm 2, \cdots$,

where $n^{m c}$ denotes the sample number after multichannel reconstruction (the time interval between succeeding azimuth samples is $\left.1 / P R F_{\text {eff }}\right)$; int $\{\cdot / \cdot\}$ denotes the integer division. As in the conventional case the useful signal is unchanged by APC and the residual modulation phase is periodic versus the order of range ambiguity, $k$, with period $M$. The DFT of the azimuth reconstructed signal, corresponding to the range ambiguity of order $k$ is [8]:

$$
X_{k}^{r}(f)=\bar{X}_{k}^{r}(f) \otimes \operatorname{DFT}\left\{\exp \left[j \varphi_{r e s}^{m c}(n, k, M)\right]\right\},
$$

where $\bar{X}_{k}^{r}(f)$ denotes the DFT of the original (i.e. without APC) azimuth reconstructed signal, corresponding to the range ambiguity of order $k ; \otimes$ the convolution operator.

\section{APC PERFORMANCE EVALUATION}

In order to characterize the APC performance, the APC Gain, $G_{a p c}$, is defined. It is a measure of the reduction of power induced by APC on the range ambiguity of $1^{\text {st }}$ order:

$$
G_{a p c}=\int_{-B_{p} / 2}^{B_{p} / 2}\left|\bar{X}_{1}^{r}(f)\right|^{2} d f / \int_{-B_{p} / 2}^{B_{p} / 2}\left|X_{1}^{r}(f)\right|^{2} d f,
$$


where $\bar{X}_{1}^{r}(f)$ denotes the DFT of the original reconstructed range ambiguity of $1^{\text {st }}$ order; $X_{1}^{r}(f)$ the DFT of the reconstructed range ambiguity of $1^{\text {st }}$ order in presence of the APC; $B_{p} \leq N P R F$ the processed bandwidth. The square module, $|\cdot|$, of the DFT is the Power Spectral Density (PSD) of the corresponding signal. Since the range ambiguity of $1^{\text {st }}$ order produces the dominant contribution to the range ambiguous power, $G_{a p c}$ is a measure of the improvement induced by APC on the image quality.

\section{NUMERICAL RESULTS}

A reference SAR system based on a planar array antenna with $N=4 \mathrm{Rx}$ subapertures is assumed. Its parameters are summarized in Table 1 (systems \# 3). Moreover, three additional SAR systems are considered, in order to evaluate the behavior of APC versus the number of Rx channels, $N$. They are obtained from the reference system by modifying the number of receive subapertures/channels from 4 to 8,2 and 1, respectively (see systems \# 4 and \# 2, \# 1 in Table 1). Note that all the systems have the same azimuth patterns.

\begin{tabular}{|l|l|l|l|l|}
\hline \multirow{2}{*}{ Parameter } & \multicolumn{4}{|l|}{ System \# } \\
\cline { 2 - 5 } & $\mathbf{1}$ & $\mathbf{2}$ & 3(Ref.) & $\mathbf{4}$ \\
\hline Orbit height $[\mathrm{km}]$ & 520 & \multicolumn{4}{|l|}{} \\
\hline Carrier frequency [GHz] & 9.600 & & \\
\hline Rx antenna total length [m] & 3 & 6 & 12 & 24 \\
\hline $\begin{array}{l}\text { Tx antenna length [m] } \\
\text { (and Rx subapert. length) }\end{array}$ & 3 & \multicolumn{4}{|l|}{} \\
\hline No. of az. Rx channels & 1 & 2 & 4 & 8 \\
\hline PRF $[\mathrm{Hz}]$ & 5068 & 2534 & 1267 & 633.5 \\
\hline PRF $_{\text {eff }}[\mathrm{Hz}]$ & 5068 & \multicolumn{4}{|l}{} \\
\hline
\end{tabular}

Tab. 1. Parameters of the planar SAR systems.

It is assumed a uniform PRF. In order to investigate the effect of the Doppler oversampling, the APC performance has been evaluated versus the oversampling factor,

$$
\gamma=P R F_{\text {eff }} / B_{p}
$$

for different processed bandwidth values. In particular, the processing bandwidth $2316 \mathrm{~Hz} \leq B_{p} \leq 4168 \mathrm{~Hz}$, which corresponds to an oversampling factor, $1.2 \leq \gamma \leq 2.1$ (and an azimuth resolution between $1.5 \mathrm{~m}$ and $2.7 \mathrm{~m}$ ), has been considered. As backscattered signal, the echo returned from a point target is considered. The effect of the elevation pattern and geometry is neglected. In particular, it is assumed that, if the APC were not employed, the signal and the range ambiguity at the input of each receive azimuth channel would have exactly the same spectrum and power, which depends only on the two-way azimuth pattern and phase history. In this sense, it is equivalent to speak of "useful signal" or "original range ambiguity".

Figure 2 shows the $G_{a p c}$ versus $\gamma$, for the SAR systems in Table 1, when $M=2$. It shows that for the analyzed cases: (i) $0.1 \mathrm{~dB} \leq G_{a p c} \leq 3.13 \mathrm{~dB}$; (ii) for a given $N$, the $G_{a p c}$ increases with the oversampling factor, $\gamma$, (iii) the $G_{a p c}$ decreases for increasing number of channels, $N$; (iv) the sensitivity of $G_{a p c}$ to $\gamma$ decreases with increasing $N$.

These results can be justified by looking at Figures 3-5.

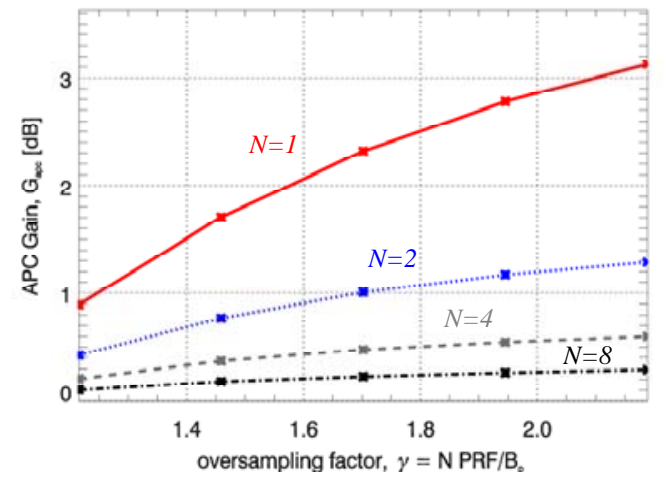

Fig. 2. $G_{a p c}(M=2)$ versus $\gamma$ for the SAR systems in Table 1 (solid line: system \# 1; dotted: \# 2; dashed: \# 3; dash-dot: \# 4).

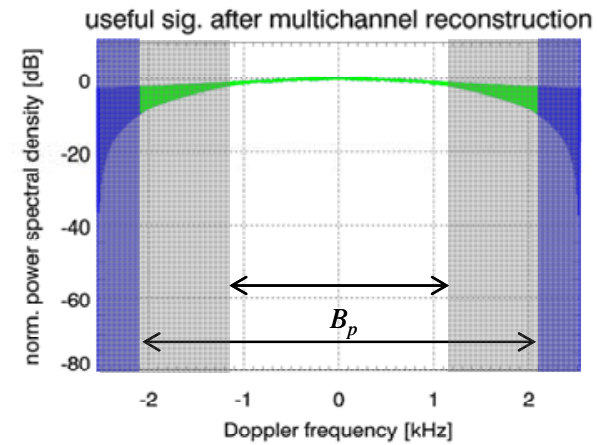

Fig. 3. Useful signal (or original $1^{\text {st }}$ order range ambiguity) PSD normalized to its maximum. $N=2$ (for $N=8$, variations are negligible), $M=2$. Shadows/arrows denote $B_{p}$ limit values.

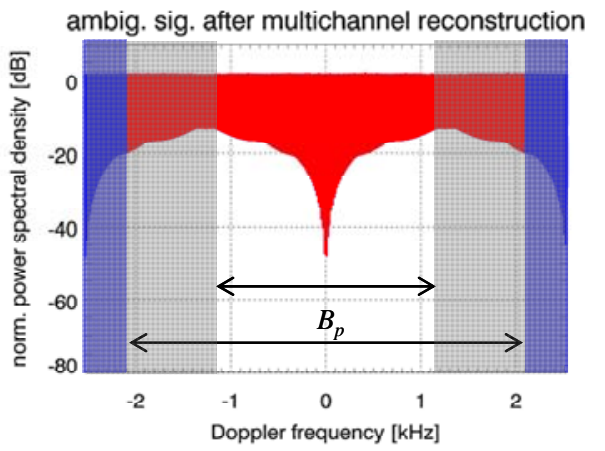

Fig. 4. Ambiguity of $1^{\text {st }}$ order PSD normalized to the max. of the PSD of the useful signal. $N=2, M=2$. 


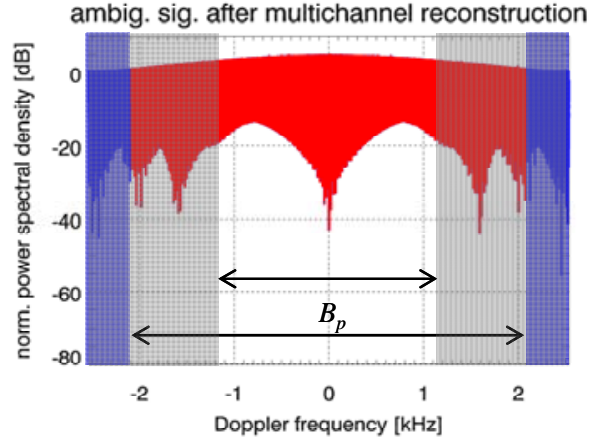

Fig. 5. Ambiguity of $1^{\text {st }}$ order PSD normalized to the max. of the PSD of the useful signal. $N=8, M=2$.

The Figures show the PSD over the $P R F_{\text {eff }}$ interval (azimuth reconstructed channel level) of the useful signal and $1^{\text {st }}$ range ambiguity, obtained in case of $N=2$ and $N=8$ : for larger $N$, the upper profile of the PSD of the signal and the ambiguity are similar and consequently $G_{a p c}$ reduces to small values, close to $0 \mathrm{~dB}$. (The thickness of the curves is a fast variation of the spectrum, due to aliasing.)

The previous analysis has been extended by considering two realistic X-band SAR multichannel systems, based on DBF on receive and SCan On REceive (SCORE) algorithm [1], employing a planar antenna and a reflector antenna, respectively. The planar system is the HRWS, promoted by the German Aerospace Centre (DLR) and conceived to obtain simultaneously high resolution and wide swaths $(1 \mathrm{~m}$ resolution, $70 \mathrm{~km}$ swath width in stripmap mode) [9]. The reflector system is an alternative design option, with the same imaging performance, currently studied in DLR [10]. Their parameters are listed in Table 2. Note that the reflector based system is characterized by a much higher oversampling. This is used in order to reduce the azimuth ambiguities resulting from the degradation of azimuth patterns at the swath edges [10].

For the HRWS based on a planar antenna, the APC Gain value obtained for $M=2$ is $0.69 \mathrm{~dB}$ : the high number of channels and the small oversampling are associated with a low $G_{a p c}$. The reflector based system, characterized by a higher oversampling factor, takes better advantage from the application of APC: $2.48 \mathrm{~dB} \leq G_{a p c} \leq 5.56 \mathrm{~dB}$ over the swath, depending on the azimuth pattern [8].

\begin{tabular}{|l|l|l|}
\hline Parameter & Planar & Reflector \\
\hline Orbit height [km] & 520 & 745 \\
\hline Carrier frequency [GHz] & 9.600 & 9.650 \\
\hline Tx/Rx antenna total length [m] & 8.75 & \\
\hline Paraboloid diameter (elev., az.) [m] & & 10,12 \\
\hline Total number of feeds (elev., az.) & & 58,10 \\
\hline No. of az. Rx channels & 7 & 10 \\
\hline PRF [Hz] & 1750 & 2450 \\
\hline Processed bandwidth [Hz] & 6252 & 5955 \\
\hline Oversampling factor & 1.960 & 4.114 \\
\hline
\end{tabular}

Tab. 2. Parameters of the HRWS systems in Stripmap mode.

\section{CONCLUSIONS}

The multichannel processing affects the APC properties. In particular, the APC effect cannot be represented by a simple frequency shift of the range ambiguity. Nevertheless, also in the multichannel case, the APC allows for improved ambiguity suppression. The analysis shows that the azimuth pattern strongly affects the APC performance. For a given azimuth pattern and resolution, the suppression is directly proportional to the oversampling factor, $\gamma$, and inversely proportional to the number of receive channels. In a conventional SAR system with $\gamma=2$, the achievable suppression of each ambiguity of odd order is about $3 \mathrm{~dB}$. In multichannel systems based on planar antenna architectures, the suppression is generally poorer. Reflector based systems are expected to reach better performance, because of the higher oversampling. For instance, in the planar and reflector based HRWS systems the APC suppression is about $0.7 \mathrm{~dB}$ and between 2.5 and $5.5 \mathrm{~dB}$, respectively. However, regardless of the actual ambiguity suppression, it could be reasonable to recur to APC, given its low implementation complexity and the increased flexibility in the SAR system design that it offers.

\section{REFERENCES}

[1] M. Suess, B. Grafmueller, R. Zahn, "A novel high resolution, wide swath SAR system," Proc. of IGARSS, vol. 3, pp. 1013-1015, July 2001.

[2] G. Krieger, N. Gebert, A. Moreira, "Multidimensional Waveform Encoding: A New Digital Beamforming Technique for Synthetic Aperture Radar Remote Sensing", IEEE Trans. on Geosci. and Remote Sens., vol. 46, no. 1, pp.31-46, Jan. 2008.

[3] S. Huber, M. Younis, A. Patyuchenko, G. Krieger, "Digital Beam Forming Techniques for Spaceborne Reflector SAR Systems". Proc. of EUSAR, pp. 962-965, June 2010.

[4] A. Moreira, "Suppressing the Azimuth Ambiguities in Synthetic Aperture Radar Images", IEEE Trans. on Geosci. and Remote Sens., vol. 31, no. 4, pp. 885 - 895, July 1993.

[5] J. Dall, A. Kusk, "Azimuth Phase Coding for Range Ambiguity Suppression in SAR", Proc. of IGARSS, vol. 3 pp. 1734-1737, Sept. 2004.

[6] N. Gebert, G. Krieger, A. Moreira, "Digital Beamforming on Receive: Techniques and Optimization Strategies for HighResolution Wide-Swath SAR Imaging", IEEE Trans. Aerosp. Electron. Syst., vol. 45, no. 2, pp. 564-592, Apr. 2009.

[7] D. Cristallini, M. Sedehi, P. Lombardo, "Imaging Solution Based on Azimuth Phase Coding." Proc. of EUSAR, pp. 57-60, June 2008

[8] F. Bordoni, M. Younis, G. Krieger, "Ambiguity Suppression by Azimuth Phase Coding in Multichannel SAR Systems", submit. to IEEE Trans. Geosc. and Remote Sensing, March 2011.

[9] F. Bordoni, M. Younis, G. Krieger, C. Fischer, "Performance Investigation on the High-Resolution Wide-Swath SAR System with Monostatic Architecture", Proc. of EUSAR, pp.1122-1125, June 2010.

[10] M. Younis, A. Patyuchenko, S. Huber, G. Krieger, A. Moreira, "A Concept for a High Performance Reflector-Based XBand SAR", Proc. of EUSAR, pp. 740-743, June 2010. 